TRANSACTIONS OF THE

AMERICAN MATHEMATICAL SOCIETY

Volume 354, Number 9, Pages 3555-3571

S 0002-9947(02)03029-5

Article electronically published on April 30, 2002

\title{
ON THE ASYMPTOTIC STABILITY FOR NONAUTONOMOUS FUNCTIONAL DIFFERENTIAL EQUATIONS BY LYAPUNOV FUNCTIONALS
}

\author{
LÁSZLÓ HATVANI
}

\begin{abstract}
Sufficient conditions are given for the asymptotic stability and uniform asymptotic stability of the zero solution of the nonautonomous FDE's whose right-hand sides can be unbounded functions of the time. The theorems are based upon Lyapunov-Krasovskiu functionals whose derivatives with respect to the equations are negative semidefinite and can vanish at long intervals. The functionals and their derivatives are estimated by either $x(t)$, the norm of the instantaneous value of the solutions or $\left\|x_{t}\right\|_{2}$, the $L_{2}$-norm of the phase segment of the solutions. Examples are given to show that the conditions are sharp, and the main theorems with the two different types of estimates are independent and improve earlier results. The theorems are applied to linear and nonlinear retarded FDE's with one delay and with distributed delays.
\end{abstract}

\section{INTRODUCTION}

Consider the nonautonomous system of functional differential equations with a finite delay

$$
x^{\prime}(t)=F\left(t, x_{t}\right),
$$

where we use the standard notation $\left[9 . C=C\left([-h, 0] ; \mathbb{R}^{n}\right)\right.$ denotes the space of the continuous functions from $[-h, 0]$ into $\mathbb{R}^{n} ; 0<h=$ const. is the size of the delay. If $x:\left[t_{0}-h, T\right) \rightarrow \mathbb{R}^{n}$ is an arbitrary function $\left(0 \leq t_{0}<T\right)$, then for any $t \in\left[t_{0}, T\right)$ we denote by $x_{t}(\in C)$ the segment of $x$ at $t$ defined by $x_{t}(u):=x(t+u)$ $(-h \leq u \leq 0)$. The right-hand side $F: \mathbb{R}_{+} \times C \rightarrow \mathbb{R}^{n}\left(\mathbb{R}_{+}:=[0, \infty)\right)$ is supposed to be continuous, so that for each $\left(t_{0}, \varphi\right) \in \mathbb{R}_{+} \times C$ there exists a solution $x\left(t_{0}, \varphi\right)$ satisfying the initial condition $x_{t_{0}}\left(t_{0}, \varphi\right)=\varphi$. We denote by $x(t)=x\left(t ; t_{0}, \varphi\right)$ the value of a solution $x\left(t_{0}, \varphi\right)$ at $t$. We will use two norms in the space $C$ :

$$
\|\varphi\|_{\infty}:=\max _{-h \leq u \leq 0}|\varphi(u)|, \quad\|\varphi\|_{2}:=\left(\int_{-h}^{0}|\varphi|^{2}(u) d u\right)^{1 / 2} \quad(\varphi \in C),
$$

where $|\cdot|$ denotes an arbitrary norm in $\mathbb{R}^{n}$. For $0<\alpha=$ const., the ball $C_{\alpha}$ in $C$ is defined by $C_{\alpha}:=\left\{\varphi \in C:\|\varphi\|_{\infty}<\alpha\right\}$.

We suppose that $F(t, 0) \equiv 0\left(t \in \mathbb{R}_{+}\right)$. This implies that the identically zero function is a solution of (1.1); it will be called the zero solution of (1.1). This

Received by the editors November 5, 2001.

2000 Mathematics Subject Classification. Primary 34K20.

The author was supported by the Hungarian National Foundation for Scientific Research (OTKA T/029188). 
paper is concerned with sufficient conditions for different kinds of stability of the zero solution of (1.1).

Definition 1.1 (see, e.g., 9]). The zero solution of (1.1) is called:

1. stable if for every $\varepsilon>0$ and $t_{0} \in \mathbb{R}_{+}$there is a $\delta\left(\varepsilon, t_{0}\right)>0$ such that $\|\varphi\|_{\infty}<\delta\left(\varepsilon, t_{0}\right)$ implies $\left|x\left(t ; t_{0}, \varphi\right)\right|<\varepsilon$ for all $t \geq t_{0}$;

2. uniformly stable if it is stable with $\delta(\varepsilon)$ independent of $t_{0}$;

3. attractive if for every $t_{0} \in \mathbb{R}_{+}$there is a $\sigma\left(t_{0}\right)>0$ such that $\|\varphi\|_{\infty}<\sigma\left(t_{0}\right)$ implies

$$
\lim _{t \rightarrow \infty} x\left(t ; t_{0}, \varphi\right)=0
$$

4. uniformly attractive if this convergence is uniform with respect to $t_{0}$ and $\varphi$; i.e., there exists a constant $\sigma>0$ and, for every $\eta>0$, there is a $T(\eta)$ such that $t \geq t_{0}+T(\eta)$ implies $\left|x\left(t ; t_{0}, \varphi\right)\right|<\eta$ for all $t_{0} \in \mathbb{R}_{+}, \varphi \in C_{\sigma}$;

5. asymptotically stable (AS for short) if it is stable and attractive;

6. uniformly asymptotically stable (UAS for short) if it is uniformly stable and uniformly attractive.

We will give sufficient conditions for AS and UAS by Lyapunov functionals. A continuous functional $V: \mathbb{R}_{+} \times C \rightarrow \mathbb{R}_{+}$is called a Lyapunov functional if its derivative $V^{\prime}$ with respect to (1.1) is not positive; i.e.,

$$
V^{\prime}(t, \varphi)=V_{\underline{\underline{1.1}}}^{\prime}(t, \varphi):=\limsup _{\gamma \rightarrow 0+}\left(\frac{1}{\gamma}\left[V\left(t+\gamma, x_{t+\gamma}(t, \varphi)\right)-V(t, \varphi)\right]\right) \leq 0 .
$$

Considering nonautonomous functional differential equations (FDE's for short), in 4, 5. we initiated the use of Lyapunov functionals whose derivatives satisfy one of the inequalities

$$
\begin{array}{rlrl}
V^{\prime}(t, \varphi) & \leq-\eta(t) W(|\varphi(0)|) & ((t, \varphi) & \left.\in \mathbb{R}_{+} \times C\right), \\
V^{\prime}(t, \varphi) & \leq-\eta(t) W\left(\|\varphi\|_{2}\right) & \left((t, \varphi) \in \mathbb{R}_{+} \times C\right) .
\end{array}
$$

Here, and in the sequel, $W$ (or $W_{1}, W_{2}, \ldots$ ) denotes a wedge, i.e., a continuous, strictly increasing function $W: \mathbb{R}_{+} \rightarrow \mathbb{R}_{+}$with $W(0)=0$, and $\eta: \mathbb{R}_{+} \rightarrow \mathbb{R}_{+}$ denotes a non-negative measurable function. As will be illuminated by examples, the coefficient $\eta$ appears in the estimates because (1.1) is nonautonomous. It may vanish on subsets in $\mathbb{R}_{+}$, so the derivative $V^{\prime}$ of the Lyapunov functional $V$ is negative semi-definite (not negative definite). Zhang [27] proved AS and UAS by the use of Lyapunov functionals whose derivatives satisfy one of the inequalities

$$
\begin{array}{ll}
V^{\prime}(t, \varphi) \leq-W(|\varphi(0)|) & \left(t \in \bigcup_{i=1}^{\infty}\left[t_{i}-h, t_{i}\right], \varphi \in C\right), \\
V^{\prime}(t, \varphi) \leq-W\left(\|\varphi\|_{2}\right) & \left(t \in \bigcup_{i=1}^{\infty}\left[t_{i}-\kappa, t_{i}\right], \varphi \in C\right)
\end{array}
$$

with appropriate sequences $\left\{t_{i}\right\}_{i=1}^{\infty}\left(\lim _{i \rightarrow \infty} t_{i}=\infty\right)$ and constant $\kappa>0$. The Lyapunov functionals were supposed to satisfy also an estimate

$$
W_{1}(|\varphi(0)|) \leq V(t, \varphi) \leq W_{2}(|\varphi(0)|)+W_{3}\left(\|\varphi\|_{2}\right)
$$

introduced by Krasovskiı [17].

In this paper we give sufficient conditions for the coefficient $\eta$ so that the combination of (1.6) and one of (1.2)-(1.3) implies AS or UAS. This theory will unify, 
simplify, complete, and improve our results 4, 15, 10, 11] and those of other papers [6, 7, 9 12, 15, 19, 25, 26, 27]. For example, Zhang's theorems [27] based on the inequality (1.4) or (1.5) can be obtained by the choice

$$
\eta(t):= \begin{cases}1 & \text { if } t \in \bigcup_{i=1}^{\infty}\left[t_{i}-h, t_{i}\right] \\ 0 & \text { otherwise }\end{cases}
$$

or

$$
\eta(t):= \begin{cases}1 & \text { if } t \in \bigcup_{i=1}^{\infty}\left[t_{i}-\kappa, t_{i}\right] \\ 0 & \text { otherwise }\end{cases}
$$

respectively.

The first part of Section 2 summarizes the preliminaries and motivates the investigation by the example of an FDE with distributed delays:

$$
x^{\prime}(t)=-a(t) x(t)+b(t) \int_{t-h}^{t} x(s) d s,
$$

where the functions $a, b: \mathbb{R}_{+} \rightarrow \mathbb{R}$ are continuous and $a(t) \geq 0$ for all $t \in \mathbb{R}_{+}$. In the second part of Section 2 we formulate and discuss the main theorems. Section 3 contains the proofs. In Section 4 we give some applications, and construct two examples showing that (1.3) is stronger than (1.2) with the same coefficient $\eta$, and that the main theorems based upon (1.2) and (1.3), respectively, are independent.

\section{Preliminaries AND Main theorems}

Lyapunov's direct method is the most effective tool of stability investigations also for FDE's. Searching for conditions of AS, Krasovskiur [17, §31] defined the negative definiteness of the derivative of a Lyapunov functional $V$ in the form

$$
V^{\prime}\left(t, x_{t}\right) \leq-W(|x(t)|) \quad(t \geq 0) .
$$

By the fundamental theorem on UAS for FDE's [17, §31] (see also [9, Th. 2.1 in Ch. 5]), condition (2.1) together with positive definiteness and decrescency of $V$ (the latter means that $V(t, \varphi) \rightarrow 0$ uniformly with respect to $t \in \mathbb{R}_{+}$as $\|\varphi\|_{\infty} \rightarrow 0$ ) imply UAS, provided that

$$
\text { for every bounded set } K \subset C \text { the image } F\left(\mathbb{R}_{+} \times K\right) \text { is bounded. }
$$

Consider the scalar nonautonomous FDE with distributed delays (1.9), and define the Lyapunov functional

$$
V(t, \varphi):=|\varphi(0)|+\int_{-h}^{0} \int_{t+u}^{t}|b(s-u)||\varphi(s-t)| d s d u .
$$

The derivative $V_{(1.9)}^{\prime}$ can be estimated as follows:

$$
\begin{aligned}
V_{[1.9)}^{\prime}\left(t, x_{t}\right) \leq & -a(t)|x(t)|+|b(t)| \int_{t-h}^{t}|x(s)| d s \\
& +\int_{-h}^{0}|b(t-u)||x(t)| d u-\int_{-h}^{0}|b(t)||x(t+u)| d u \\
=- & {\left[a(t)-\int_{t}^{t+h}|b(s)| d s\right]|x(t)| . }
\end{aligned}
$$

Applying the fundamental theorem on UAS, we obtain: if 
1. there exists a constant $\gamma$ such that

$$
a(t)-\int_{t}^{t+h}|b(s)| d s \geq \gamma>0 \quad\left(t \in \mathbb{R}_{+}\right)
$$

2. functions $a, b$ are bounded on $\mathbb{R}_{+}$,

then the zero solution of (1.9) is UAS.

If (1.9) is autonomous, i.e., $a(t) \equiv a_{0}=$ const. and $b(t) \equiv b_{0}=$ const., this consequence says that $a_{0}>\left|b_{0}\right| h$ implies UAS. This sufficient condition is sharp in the sense that, in the case $a_{0}=b_{0} h$, every constant function is a solution of (1.9); so the zero solution is not AS. However, if (1.9) is nonautonomous, then 1 and 2 are not sharp for two reasons. In the first place, obviously, the larger $a(t)$ the better from the point of view of UAS; so the boundedness of $a$ is not natural. In the second place, condition 1 seems too strong: one is convinced that UAS can be proved also if $V$ is driven to zero "intermittently", i.e., if the coefficient $a(t)-\int_{t}^{t+h}|b|$ vanishes over whole intervals. These observations show that the fundamental theorem is still strongly connected to autonomous systems and cannot handle some important really nonautonomous cases. This can already be seen from the definition of positive definiteness: the right-hand side of (2.1) may not depend on the time $t$ explicitly. Besides, AS and UAS are different properties for nonautonomous systems. This gives rise to a third problem: what conditions imply AS (maybe not uniform)?

The first problem (about the boundedness of $a$ in (1.9)) is caused by condition (2.2). Kato [13] and Makay [21, 22] showed by examples that this condition cannot be dropped from the fundamental theorem; namely, the rest of the conditions do not imply, in general, even AS. However, Krasovskiı̌ [17, Theorem 31.3] has discovered that AS can be proved after dropping condition (2.2) if the decrescency condition is strengthened in the following way:

$$
V(t, \varphi) \leq W_{2}(|\varphi(0)|)+W_{3}\left(\|\varphi\|_{2}\right) \quad\left((t, \varphi) \in \mathbb{R}_{+} \times C\right) .
$$

Later on, Burton [3] proved UAS under the same conditions.

Apply condition (2.6) to the Lyapunov functional (2.3). Changing the order of integration and using Schwarz's inequality, we get

$$
\begin{aligned}
\int_{-h}^{0} \int_{t+u}^{t}|b(s-u)||\varphi(s-t)| d s d u & =\int_{t-h}^{t} \int_{-h}^{s-t}|b(s-u)| d u|\varphi(s-t)| d s \\
& \leq\left[\int_{t-h}^{t}\left(\int_{t}^{s+h}|b(r)| d r\right)^{2} d s\right]^{1 / 2}\|\varphi\|_{2},
\end{aligned}
$$

whence we obtain: if

1. there exists a constant $\gamma$ such that (2.5) is satisfied, and

2. there is a constant $M_{1}$ such that

$$
\int_{t-h}^{t}\left(\int_{t}^{s+h}|b(r)| d r\right)^{2} d s \leq M_{1} \quad\left(t \in \mathbb{R}_{+}\right)
$$

then the zero solution of (1.9) is UAS.

This consequence shows that using $\|\cdot\|_{2}$ in the decrescency condition (2.6) made it possible to get rid of the boundedness condition (2.2), and this solved our problem with the boundedness of coefficient $a$ in Equation (1.9). We will establish generalizations of the Krasovskiı-Burton Theorem solving the other two problems raised 
above. First, however, let us point out another direction of the further development of the theory. It has been observed [4, 5, 10] 21, 25, 26, 27] that using the $\|\cdot\|_{2}$ norm also in condition (2.1) about the negative definiteness of $V^{\prime}$ makes these theorems more effective. This can be illuminated by the example of Equation (1.9) with the Lyapunov functional

$$
V(t, \varphi):=|\varphi(0)|+\beta \int_{-h}^{0} \int_{t+u}^{t}|b(s-u)||\varphi(s-t)| d s d u \quad(1<\beta=\text { const. })
$$

whose derivative with respect to Equation (1.9) can be estimated by

$$
V_{\text {[1.9] }}^{\prime}\left(t, x_{t}\right) \leq-\left[a(t)-\beta \int_{t}^{t+h}|b|\right]|x(t)|-(\beta-1)|b(t)| \int_{t-h}^{t}|x(u)| d u
$$

instead of (2.4).

Having been motivated by such examples, in [4, 5] we initiated the use of Lyapunov functionals whose derivatives satisfy (1.2) or (1.3). If we want to deduce AS from these inequalities, then, obviously, we have to require $\int_{0}^{\infty} \eta=\infty$. It will be pointed out in Section 4 that in the case of (1.2) this is not sufficient: we have to require also the divergence $\int_{H} \eta=\infty$ over every subset $H \subset \mathbb{R}_{+}$dense enough in $\mathbb{R}_{+}$.

Definition 2.1. A subset $H \subset \mathbb{R}_{+}$is called $h$-dense on the interval $\left[t_{0}, \infty\right)$ if there exists a constant $\kappa>0$ such that

$$
\mu([t, t+h] \cap H) \geq \kappa \text { for all } t \geq t_{0},
$$

where $\mu(\cdot)$ denotes the Lebesgue measure.

Theorem 2.2. Suppose that there exist a continuous functional $V: \mathbb{R}_{+} \times C \rightarrow \mathbb{R}_{+}$, wedges $W_{1}, W_{2}, W_{3}, W$, and a measurable function $\eta: \mathbb{R}_{+} \rightarrow \mathbb{R}_{+}$satisfying the conditions

(i) $W_{1}(|x(t)|) \leq V\left(t, x_{t}\right)$,

(ii) $V\left(t, x_{t}\right) \leq W_{2}(|x(t)|)+W_{3}\left(\left\|x_{t}\right\|_{2}\right)$,

(iii) $V^{\prime}\left(t, x_{t}\right) \leq-\eta(t) W(|x(t)|)$

along every solution $x:\left[t_{0}, \infty\right) \rightarrow \mathbb{R}^{n}$ of Equation (1.1) for all $t \geq t_{0}$.

A. If for every $t_{0} \in \mathbb{R}_{+}$and every $h$-dense subset $H \subset\left[t_{0}, \infty\right)$ the divergence

$$
\lim _{K \rightarrow \infty} \int_{\left[t_{0}, t_{0}+K\right] \cap H} \eta(t) d t=\infty
$$

holds, then the zero solution of Equation (1.1) is AS.

B. Suppose that the divergence in (2.10) is uniform with respect to $t_{0}$ and $H$, i.e., for every $L$ there is an $N=N(L)$ such that $K>N(L)$ implies $\int_{\left[t_{0}, t_{0}+K\right] \cap H} \eta(t) d t>$ $L$ for all $t_{0} \in \mathbb{R}_{+}$and for all subsets $H \subset \mathbb{R}_{+} h$-dense on $\left[t_{0}, \infty\right)$. Then the zero solution of Equation (1.1) is UAS.

Condition (2.10) allows the coefficient $\eta$ to vanish on whole intervals of whatever length. For example, consider the step functions

$$
\begin{aligned}
& \eta_{1}(t):= \begin{cases}1 & \text { if } t \in \bigcup_{k \in \mathbb{N}}[3 k h,(3 k+1) h], \\
0 & \text { otherwise }\end{cases} \\
& \eta_{2}(t):= \begin{cases}1 & \text { if } t \in \bigcup_{k \in \mathbb{N}}\left[k^{2} h,\left(k^{2}+1\right) h\right], \\
0 & \text { otherwise. }\end{cases}
\end{aligned}
$$


It is easy to see that both $\eta_{1}$ and $\eta_{2}$ satisfy (2.10); namely, the divergence in (2.10) with $\eta_{1}$ is uniform with respect to $t_{0}, H$. However, if we define

$$
\eta_{3}(t):= \begin{cases}1 & \text { if } t \in \bigcup_{k \in \mathbb{N}}[k h,(k+1 / 2) h], \\ 0 & \text { otherwise }\end{cases}
$$

then $\eta_{3}$ does not satisfy condition (2.10), because the set

$$
H:=\bigcup_{k=0}^{\infty}\left(\left(k+\frac{1}{2}\right) h,(k+1) h\right)
$$

is $h$-dense on $[0, \infty)$ (with $\kappa=h / 2$ ); nevertheless, $\int_{H} \eta_{3}=0 \neq \infty$.

It is worth noticing that condition (2.10) and $\int_{0}^{\infty} \eta=\infty$ are equivalent for every nonincreasing function $\eta$. In fact, if $\int_{0}^{\infty} \eta=\infty$ and $H \subset \mathbb{R}_{+}$is $h$-dense on $\left[t_{0}, \infty\right)$ with constant $\kappa$, then

$$
\int_{H} \eta(t) d t \geq \sum_{k=\left[t_{0} / h\right]+1}^{\infty} \int_{[k h,(k+1) h] \cap H} \eta(t) d t \geq \kappa \sum_{k=\left[t_{0} / h\right]+1}^{\infty} \eta((k+1) h)=\infty,
$$

which means that (2.10) is satisfied ([.] denotes the greatest integer function). On the other hand, if (2.10) is satisfied, then $\int_{0}^{\infty} \eta=\infty$, because interval $[0, \infty)$ is an $h$-dense set on $[0, \infty)$ (with constant $\kappa=h$ ).

This shows that condition (2.10) coincides with the best possible one $\int_{0}^{\infty} \eta=\infty$ for monotone coefficients $\eta$. The next theorem says that $\int_{0}^{\infty} \eta=\infty$ is sufficient for arbitrary $\eta$ if we have (1.3) instead of (1.2).

Theorem 2.3. Suppose that there exist a continuous functional $V: \mathbb{R} \times C \rightarrow$ $\mathbb{R}_{+}$, wedges $W_{1}, W_{2}, W_{3}, W$, and a measurable function $\eta: \mathbb{R}_{+} \rightarrow \mathbb{R}_{+}$satisfying conditions (i) and (ii) in Theorem 2.2, and

(iii) $V^{\prime}\left(t, x_{t}\right) \leq-\eta(t) W\left(\left\|x_{t}\right\|_{2}\right)$

along every solution $x:\left[t_{0}, \infty\right) \rightarrow \mathbb{R}^{n}$ of Equation (1.1) for all $t \geq t_{0}$.

A. If for every $t_{0} \in \mathbb{R}_{+}$the divergence

$$
\lim _{K \rightarrow \infty} \int_{t_{0}}^{t_{0}+K} \eta(t) d t=\infty
$$

holds, then the zero solution of Equation (1.1) is AS.

B. If the divergence in (2.11) is uniform with respect to $t_{0} \in \mathbb{R}_{+}$, then the zero solution of Equation (1.1) is UAS.

In order to illuminate their scope, we apply our theorems to the test equation (1.9) using Lyapunov functionals (2.3) and (2.8), respectively:

1. If (2.7) is satisfied, $a(t)-\int_{t}^{t+h}|b| \geq 0$ for all $t \in \mathbb{R}_{+}$, and for every $t_{0} \in \mathbb{R}_{+}$,

$$
\int_{\left[t_{0}, \infty\right) \cap H}\left[a(t)-\int_{t}^{t+h}|b|\right] d t=\infty \text { for all } h \text {-dense sets } H \subset \mathbb{R}_{+},
$$

then the zero solution of Equation (1.9) is AS. If the divergence in (2.12) is uniform with respect to $t_{0} \in \mathbb{R}_{+}$and $H \subset \mathbb{R}_{+}$, then the zero solution is UAS.

2. If (2.7) is satisfied, there exists a $\beta>1$ such that $a(t)-\beta \int_{t}^{t+h}|b| \geq 0$ for all $t \in \mathbb{R}_{+}$, and

$$
\int_{t_{0}}^{\infty}|b(t)| d t=\infty
$$


then the zero solution of (1.9) is AS. If the divergence in (2.13) is uniform with respect to $t_{0} \in \mathbb{R}_{+}$, then the zero solution is UAS.

Remark 2.4. It should be observed that these statements are not independent. Condition (2.12) can be satisfied without fulfilment of (2.13). On the other hand, however, condition (2.13) together with the existence of $\beta$ implies (2.12). In Section 4 we construct an equation and a Lyapunov functional such that Theorem 2.3 is applicable but Theorem 2.2 is not. So it will be shown that our two fundamental theorems are independent.

Remark 2.5. Zhang [27] proved the following two statements: Suppose that conditions (i) and (ii) in Theorem 2.2 are satisfied.

1. If condition (1.4) holds with some sequence $\left\{t_{i}\right\}_{i=1}^{\infty}\left(\lim _{i \rightarrow \infty} t_{i}=\infty\right)$, then the zero solution of Equation (1.1) is AS. If, in addition, there is a constant $J>0$ such that $t_{i+1}-t_{i} \leq J(i \in \mathbb{N})$, then the zero solution is UAS.

2. If condition (1.5) holds with some sequence $\left\{t_{i}\right\}_{i=1}^{\infty}\left(\lim _{i \rightarrow \infty} t_{i}=\infty\right)$ and constant $\kappa>0$, then the zero solution of (1.1) is AS. If, in addition, there is a constant $J>0$ with the same property as in the previous statement, then the zero solution is UAS.

Obviously, these statements are consequences of Theorems 2.2 and 2.3 it is enough to make the choices (1.7) and (1.8), respectively. On the other hand, our theorems are more general. For example, let us define the saw-shaped function

$$
\eta_{1}(t):= \begin{cases}t-(k-1) h & \text { if }(k-1) h \leq t \leq(k-1 / 2) h, \\ -t+k h & \text { if }(k-1 / 2) h<t<k h, k \in \mathbb{N},\end{cases}
$$

and the step function

$$
\eta_{2}(t):= \begin{cases}k^{2} & \text { if } k \leq t \leq k+1 / k^{2}, k \in \mathbb{N}, \\ 0 & \text { otherwise }\end{cases}
$$

for conditions (1.2) and (1.3), respectively. Then (2.10) and (2.11) are satisfied uniformly with coefficients $\eta_{1}$ and $\eta_{2}$, respectively; so Theorems 2.2 and 2.3 yield UAS. On the other hand, the sequence $\left\{t_{i}\right\}_{i=1}^{\infty}$ and the constant $\kappa>0$ cannot be chosen so that (1.2) implies (1.4), or (1.3) implies (1.5). Therefore, in both cases, Zhang's theorems cannot guarantee even AS.

Remark 2.6. In 4, considering more general situations, we already proved sufficient conditions for UAS based upon other properties of the coefficients $\eta$ (integral positivity, positivity in measure, ... ). These properties were used by other authors 1, 2, 8, 14, 15 16, 18, 19, 23, 24 20, 25, 26. Parts B in Theorems 2.2 and 2.3 show that these conditions can be essentially weakened if the Lyapunov functional is decrescent in Krasovski1's sense. The method of proof brings also assumptions for AS at the same time; so we included parts A, proved in [5] by other methods.

\section{Proofs}

The first lemma gives an estimate from below for the Lebesgue measure of a level set of a $\varphi \in C$ in terms of $\|\varphi\|_{\infty}$ and $\|\varphi\|_{2}$.

Lemma 3.1. For $\varphi \in C_{M}$ and $\kappa \in(0, M)$, introduce the notation

$$
P(\varphi, \kappa):=\{u \in[-h, 0]:|\varphi(u)| \geq \kappa\} .
$$


Then

$$
\mu\left(P\left(\varphi,\|\varphi\|_{2} / \sqrt{2 h}\right)\right) \geq\|\varphi\|_{2}^{2} / 2 M^{2} .
$$

Proof. We have

$$
\|\varphi\|_{2}^{2}=\int_{-h}^{0}|\varphi(u)|^{2} d u \leq M^{2} \mu(P(\varphi, \kappa))+\kappa^{2}[h-\mu(P(\varphi, \kappa))],
$$

whence

$$
\mu(P(\varphi, \kappa)) \geq \frac{\|\varphi\|_{2}^{2}-\kappa^{2} h}{M^{2}-\kappa^{2}} .
$$

Choose $\kappa:=\|\varphi\|_{2} / \sqrt{2 h}$. Then $\kappa^{2}<M^{2}$, and

$$
\mu\left(P\left(\varphi,\|\varphi\|_{2} / \sqrt{2 h}\right)\right) \geq \frac{\|\varphi\|_{2}^{2}-\frac{\|\varphi\|_{2}^{2}}{2 h} h}{M^{2}}=\frac{\|\varphi\|_{2}^{2}}{2 M^{2}} .
$$

Proof of Theorem 2.2, Conditions (i) and (ii) imply uniform stability (see, e.g., 9, Th. 5.2.1]), which means that for every $\varepsilon>0$ there is a $\delta(\varepsilon)>0$ such that $\|\varphi\|_{\infty}<\delta(\varepsilon)$ implies $\left|x\left(t ; t_{0}, \varphi\right)\right|<\varepsilon$ for all $t_{0} \in \mathbb{R}_{+}, t \geq t_{0}$. Take $\sigma:=\delta(1)>$ 0 , and consider an arbitrary solution $x\left(t_{0}, \varphi\right)$ with $\|\varphi\|_{\infty}<\sigma$. We know that $\left|x\left(t ; t_{0}, \varphi\right)\right|<1$ for all $t \geq t_{0}$. Introduce the notation $v(t):=V\left(t, x_{t}\left(t_{0}, \varphi\right)\right)$. This is a non-negative, non-increasing function bounded above by the quantity

$$
V\left(t_{0}, \varphi\right) \leq W_{2}(\sigma)+W_{3}(\sigma \sqrt{h})=: c_{1},
$$

independent of $t_{0}$ and $\varphi$.

To prove AS, for every $\gamma>0, t_{0} \in \mathbb{R}_{+}$, and $\varphi \in C_{\sigma}$, we have to find $T=$ $T\left(\gamma, x\left(t_{0}, \varphi\right)\right)$ such that $\left|x\left(t ; t_{0}, \varphi\right)\right|<\gamma$ holds for all $t \geq t_{0}+T$. To this end it is sufficient to know that $T>h$, and

$$
v\left(t_{0}+T-h\right)=V\left(t_{0}+T-h, x_{t_{0}+T-h}\right)<W_{1}(\delta(\gamma))=: c_{2}(\gamma)
$$

is satisfied. In fact, then

$$
W_{1}(|x(s)|) \leq V\left(s, x_{s}\right) \leq V\left(t_{0}+T-h, x_{t_{0}+T-h}\right)<W_{1}(\delta(\gamma)),
$$

i.e., $|x(s)|<\delta(\gamma)$ for all $s \in\left[t_{0}+T-h, t_{0}+T\right]$, which means that $\left\|x_{t_{0}+T}\right\|_{\infty}<\delta(\gamma)$. Since $\delta(\gamma)$ is the constant of uniform stability, we have $|x(t)|<\gamma$ for all $t \geq t_{0}+T$.

First we prove AS by showing the existence of $T=T\left(\gamma, x\left(t_{0}, \varphi\right)\right)$ with property (3.2) under condition (2.10). Then we observe that the uniform version of (2.10) implies that $T=T(\gamma)$ is independent of the solution $x\left(t_{0}, \varphi\right)$, which means UAS.

For fixed $\gamma>0, t_{0} \in \mathbb{R}_{+}, \varphi \in C_{\sigma}$, solution $x\left(t_{0}, \varphi\right)$, and $K>0$ let us define the sets

$$
\begin{aligned}
& H_{0}^{K}=H_{0}^{K}\left(\gamma, x\left(t_{0}, \varphi\right)\right):=\left\{t \in\left[t_{0}, t_{0}+K\right]:|x(t)|>W_{2}^{-1}\left(\frac{c_{2}(\gamma)}{3}\right)=: c_{3}(\gamma)\right\}, \\
& H_{2}^{K}=H_{2}^{K}\left(\gamma, x\left(t_{0}, \varphi\right)\right):=\left\{t \in\left[t_{0}, t_{0}+K\right]:\left\|x_{t}\right\|_{2}>W_{3}^{-1}\left(\frac{c_{2}(\gamma)}{3}\right)=: c_{4}(\gamma)\right\} .
\end{aligned}
$$

If we cannot find $T \leq K+h$ with (3.2), then $H_{0}^{K}$ and $H_{2}^{K}$ together cover the interval $\left[t_{0}, t_{0}+K\right]$, because otherwise we would have $t_{*} \in\left[t_{0}, t_{0}+K\right]$ with $\left|x\left(t_{*}\right)\right| \leq c_{3}$, 
$\left\|x_{t_{*}}\right\|_{2} \leq c_{4}$, and we would obtain

$$
\begin{aligned}
c_{2} \leq v\left(t_{0}+K\right) & \leq v\left(t_{*}\right)=V\left(t_{*}, x_{t_{*}}\left(t_{0}, \varphi\right)\right) \\
& \leq W_{2}\left(\left|x\left(t_{*}\right)\right|\right)+W_{3}\left(\left\|x_{t_{*}}\right\|_{2}\right) \leq \frac{c_{2}}{3}+\frac{c_{2}}{3}<c_{2},
\end{aligned}
$$

a contradiction. Therefore, if there is no $T$ in $\left[t_{0}, t_{0}+K+h\right]$, then $H_{0}^{K} \cup H_{2}^{K} \supset$ $\left[t_{0}, t_{0}+K\right]$.

By Lemma 3.1, if $t \in H_{2}^{K}$, then there is a set $Q(t) \subset[t-h, t]$ with $\mu(Q(t)) \geq$ $c_{4}^{2}(\gamma) / 2=: c_{5}(\gamma)$ such that

$$
|x(s)| \geq c_{4} / \sqrt{2 h} \text { for } s \in Q(t) .
$$

Let us introduce the notation

$$
Q^{K}:=\left(\bigcup_{t \in H_{2}^{K}} Q(t)\right) \backslash H_{0}^{K} .
$$

Now, we can estimate the decrease of $V$ along the solution $x\left(t_{0}, \varphi\right)$. From condition (iii), the definitions of $H_{0}^{K}, H_{2}^{K}$, and inequalities (3.1), (3.3) we get

$$
\begin{aligned}
0 \leq v\left(t_{0}+K+h\right) & \leq v\left(t_{0}\right)+\int_{t_{0}}^{t_{0}+K} v^{\prime}(t) d t \\
& \leq c_{1}+\int_{H_{0}^{K}} v^{\prime}(t) d t+\int_{Q^{K}} v^{\prime}(t) d t \\
& \leq c_{1}-c_{6} \int_{H_{0}^{K}} \eta(t) d t-c_{7} \int_{Q^{K}} \eta(t) d t \\
& \leq c_{1}-c_{8} \int_{H_{0}^{K} \cup Q^{K}} \eta(t) d t
\end{aligned}
$$

where

$$
c_{6}(\gamma):=W\left(c_{3}(\gamma)\right), c_{7}(\gamma):=W\left(\frac{c_{4}(\gamma)}{\sqrt{2 h}}\right), c_{8}(\gamma):=\min \left\{c_{6}(\gamma) ; c_{7}(\gamma)\right\} .
$$

Let us conclude the proof of AS by the method of contradiction. Suppose that the zero solution of (1.1) is not AS. Then there are $t_{0} \in \mathbb{R}_{+}, \varphi \in C_{\sigma}, x\left(t_{0}, \varphi\right)$, and $\gamma>0$ such that there exists no $T<K+h$ with (3.2) for all $K>0$. First we show that the set $H=H\left(\gamma, x\left(t_{0}, \varphi\right)\right):=\bigcup_{K>0}\left(H_{0}^{K} \cup Q^{K}\right)$ is $h$-dense in $\left[t_{0}, \infty\right)$ with the constant $\kappa=\kappa(\gamma):=c_{5}(\gamma) / 2$.

In fact, if $t \geq t_{0}+h$, then, for some $K>0$, either $\left[t-c_{5} / 2, t\right] \subset H_{0}^{K}$, or the interval $\left[t-c_{5} / 2, t\right]$ contains a point of $H_{2}^{K}$. In the latter case, the interval $\left[t-h-c_{5} / 2, t\right]$ contains a subset of $Q^{K}$ with measure $c_{5}$. Therefore, in any case we have

$$
\mu\left([t-h, t] \cap\left(H_{0}^{K} \cup Q^{K}\right)\right) \geq \frac{c_{5}}{2}=\kappa,
$$

which means that $H$ is $h$-dense in $\left[t_{0}, \infty\right)$ with the constant $\kappa$.

Finally, from condition (2.10) and the estimate (3.4) we obtain

$$
\begin{aligned}
0 \leq \lim _{t \rightarrow \infty} v(t) & \leq v\left(t_{0}\right)+\int_{t_{0}}^{\infty} v^{\prime}(t) d t \\
& \leq c_{1}-c_{8}(\gamma) \int_{H\left(\gamma, x\left(t_{0}, \varphi\right)\right)} \eta(t) d t=-\infty .
\end{aligned}
$$


This is a contradiction; hence, for every $x\left(t_{0}, \varphi\right)$ and $\gamma>0$ there is a $K\left(\gamma, x\left(t_{0}, \varphi\right)\right)$ such that (3.2) is fulfilled with some $T\left(\gamma, x\left(t_{0}, \varphi\right)\right)<K\left(\gamma, x\left(t_{0}, \varphi\right)\right)+h$; i.e., the zero solution is AS.

To prove statement B in the theorem we have to observe that the size of $K$ (and, consequently, of $T$ ) is governed only by $v\left(t_{0}\right)$ and the speed of divergence in (3.5). However, $v\left(t_{0}\right) \leq c_{1}$, and $c_{8}(\gamma)$ is independent of $x\left(t_{0}, \varphi\right)$; hence, if we require that $\int_{H} \eta$ diverge uniformly with respect to $t_{0} \in \mathbb{R}_{+}$and $h$-dense sets $H \subset \mathbb{R}_{+}$, then $T=T(\gamma)$ is independent of $t_{0}, \varphi$, and $x\left(t_{0}, \varphi\right)$; i.e., the zero solution is UAS.

Remark 3.2. This proof allows us to generalize the upper bound on $V$ in Krasovskiı's condition (2.6). Theorem 2.2 remains true after replacing condition (ii) by

(ii') $V\left(t, x_{t}\right) \leq W_{2}(\max \{|x(t-h / 2)| ;|x(t)|\})+W_{3}\left(\left\|x_{t}\right\|_{2}\right)$.

In fact, we have to modify the proof in the following way. In addition to sets $H_{0}^{K}, H_{2}^{K}$, we define also

$$
\begin{aligned}
H_{1 / 2}^{K} & =H_{1 / 2}^{K}\left(\gamma, x\left(t_{0}, \varphi\right)\right) \\
& :=\left\{t \in\left[t_{0}, t_{0}+K\right]:\left|x\left(t-\frac{h}{2}\right)\right|>W_{2}^{-1}\left(\frac{c_{2}(\gamma)}{3}\right)=c_{3}\right\} .
\end{aligned}
$$

Now if we cannot find $T \leq K$ with (3.2), then $H_{0}^{K}, H_{1 / 2}^{K}$, and $H_{2}^{K}$ together cover the interval $\left[t_{0}, t_{0}+K\right]$. On $H_{1 / 2}^{K}$ we have the estimate

$$
\begin{aligned}
\int_{H_{1 / 2}^{K}} \eta\left(t-\frac{h}{2}\right) W\left(\left|x\left(t-\frac{h}{2}\right)\right|\right) d t & \\
& \geq W\left(c_{3}\right) \int_{H_{1 / 2}^{K}} \eta\left(t-\frac{h}{2}\right) d t \geq c_{6} \int_{\left\{H_{1 / 2}^{K}-h / 2\right\}} \eta(s) d s,
\end{aligned}
$$

where the set $\left\{H_{1 / 2}^{K}-h / 2\right\} \subset \mathbb{R}_{+}$is defined as the shift of the set $H_{1 / 2}^{K}$ to the left by $h / 2$. Therefore, now we have to consider the set

$$
H:=\bigcup_{K>0}\left(H_{0}^{K} \cup\left\{H_{1 / 2}^{K}-h / 2\right\} \cup Q^{K}\right) .
$$

It is $h$-dense in $\left[t_{0}, \infty\right)$, which can be proved in the same way as in the original proof.

In Section 4 we will need this modification of Theorem 2.2.

Proof of Theorem 2.3. This coincides with that of Theorem 2.2 to the point where we applied Lemma3.1. So we suppose also here that there is no $T<K+h$ with property (3.2), whence we know that $H_{0}^{K}$ and $H_{2}^{K}$ together cover $\left[t_{0}, t_{0}+K\right]$. We will find a constant $c_{9}(\gamma)>0$ such that $\left\|x_{t}\right\|_{2} \geq c_{9}(\gamma)$ for all $t \in\left[t_{0}, t_{0}+K\right]$.

Instead of Lemma 3.1 in this proof we will use the following fact: If $t_{*} \in H_{2}^{K}$, and $\left|t-t_{*}\right|<3 c_{4}^{2} / 4$, then $\left\|x_{t}\right\|_{2}>c_{4} / 2$. In fact, $\left|\left(\left\|x_{t}\right\|_{2}^{2}\right)^{\prime}\right|=\left.|| x\right|^{2}(t)-|x|^{2}(t-h) \mid \leq 1$; therefore, $\left|\left\|x_{t}\right\|_{2}^{2}-\left\|x_{t_{*}}\right\|_{2}^{2}\right|<3 c_{4}^{2} / 4$, and we have $\left\|x_{t}\right\|_{2}^{2}>c_{4}^{2} / 4$, i.e., $\left\|x_{t}\right\|_{2}>c_{4} / 2$.

The sets $H_{0}^{K}$ and $H_{2}^{K}$ are open in $\left[t_{0}, t_{0}+K\right]$. Since $\left[t_{0}, t_{0}+K\right]$ is compact, by Borel's theorem $\left(t_{0}, t_{0}+K\right)$ can be covered by finite open intervals $\left\{I_{p}^{0}\right\}_{p=1}^{k_{0}}$ $\left(I_{p}^{0} \subset H_{0}^{K}, p=1,2, \ldots, k_{0}\right)$ and $\left\{I_{q}^{2}=\left(a_{q}^{2}, b_{q}^{2}\right)\right\}_{q=1}^{k_{2}}\left(I_{q}^{2} \subset H_{2}^{K}, q=1,2, \ldots, k_{2}\right)$. By the fact we have just proved we know that $\left\|x_{t}\right\|_{2} \geq c_{4} / 2$ for all $t \in \bigcup_{q=1}^{k_{2}} I_{q}^{2^{\prime}}$, where $I_{q}^{2^{\prime}}=\left(a_{q}^{2}-3 c_{4}^{2} / 4, b_{q}^{2}+3 c_{4}^{2} / 4\right)$. Obviously, $\left\{I_{p}^{0}\right\}_{p=1}^{k_{0}}$ and $\left\{I_{q}^{2^{\prime}}\right\}_{q=1}^{k_{2}}$ even more 
cover $\left(t_{0}, t_{0}+K\right)$. If $t_{*} \notin \bigcup_{q=1}^{k_{2}} I_{q}^{2^{\prime}}$, then $t_{*} \in H_{0}^{K}$; what is more, $|x(t)| \geq c_{3}$ holds in the interval $\left(t_{*}-3 c_{4}^{2} / 4, t_{*}\right)$. Therefore,

$$
\left\|x_{t_{*}}\right\|_{2} \geq\left(c_{3}^{2} \min \left\{h ; 3 c_{4}^{2} / 4\right\}\right)^{1 / 2}=c_{3} \min \left\{\sqrt{h} ; \frac{\sqrt{3}}{2} c_{4}\right\} .
$$

Taking into account the estimate for $\left\|x_{t}\right\|_{2}$ on $\bigcup_{q=1}^{k_{2}} I_{q}^{2^{\prime}}$, we get

$$
\left\|x_{t}\right\|_{2} \geq c_{9}(\gamma):=\min \left\{\frac{c_{4}(\gamma)}{2} ; \sqrt{h} c_{3}(\gamma) ; \frac{\sqrt{3}}{2} c_{3}(\gamma) c_{4}(\gamma)\right\} \text { for all } t \in\left[t_{0}, t_{0}+K\right] .
$$

Condition (iii) and this inequality imply

$$
\begin{aligned}
0 \leq v\left(t_{0}+K\right) & \leq v\left(t_{0}\right)-\int_{t_{0}}^{t_{0}+K} \eta(t) W\left(\left\|x_{t}\right\|_{2}\right) d t \\
& \leq c_{1}-c_{10}(\gamma) \int_{t_{0}}^{t_{0}+K} \eta(t) d t, \quad c_{10}(\gamma):=W\left(c_{9}(\gamma)\right) .
\end{aligned}
$$

By (2.11), the last estimate yields a contradiction if $K \rightarrow \infty$. This means that for every $x\left(t_{0}, \varphi\right)$ and $\gamma>0$ there is a $T\left(\gamma, x\left(t_{0}, \varphi\right)\right)$ with (3.2); i.e., the zero solution is AS.

Estimate (3.6) shows that a $T=T(\gamma)$, independent of $t_{0}, \varphi$, and the solution $x\left(t_{0}, \varphi\right)$, should exist provided that the divergence in (2.11) is uniform with respect to $t_{0} \in \mathbb{R}_{+}$. This means that the zero solution is UAS.

\section{Applichtions and examples}

4.1. Retarded FDE with one delay. Consider the equation

$$
x^{\prime}(t)=-a(t) f(x(t))+b(t) g(x(t-h)),
$$

where $a, b: \mathbb{R}_{+} \rightarrow \mathbb{R}$ are continuous, $f, g: \mathbb{R} \rightarrow \mathbb{R}$ are continuously differentiable, and $a(t) \geq 0, x f(x)>0$ for all $t \in \mathbb{R}_{+}, x \neq 0$. Suppose that there exists a constant $c>0$ such that $|g(x)| \leq c|f(x)|$ for sufficiently small $|x|$. Using the notation $F(x):=\int_{0}^{x} f(r) d r$, we introduce the Lyapunov functional

$$
V(t, \varphi):=F(\varphi(0))+\int_{t-h}^{t} \nu(s) g^{2}(\varphi(s-t)) d s
$$

with a properly chosen auxiliary function $\nu: \mathbb{R}_{+} \rightarrow \mathbb{R}_{+}$. By the inequality $\alpha x+$ $\beta x^{2} \geq-\alpha^{2} / 4 \beta$ for $x \in \mathbb{R}(\beta>0)$, the derivative of $V$ with respect to (4.1) can be estimated as follows:

$$
V_{\text {(4.11) }}^{\prime}\left(t, x_{t}\right) \leq-\left[a(t)-c^{2} \nu(t)-\frac{b^{2}(t)}{4 \nu(t-h)}\right] f^{2}(x(t)) .
$$

In [17,9] the functional (4.2) was used with $\nu(t)=\nu_{0}=$ const. chosen by Busenberg and Cooke 7 to involve varying $\nu(t)$. One of their choices was

$$
\nu(t):=(1 / 2 p) b^{2}(t+h)
$$

$(0<p=$ const. $)$, in which case the estimate (4.3) has the form

$$
V_{\text {[4.1) }}^{\prime}\left(t, x_{t}\right) \leq-\frac{1}{2 p}\left[2 p a(t)-c^{2} b^{2}(t+h)-p^{2}\right] f^{2}(x(t)) .
$$

Considering the linear case $(f(x) \equiv g(x) \equiv x, c=1)$ and using an "annulus argument", they proved the following: 
Theorem 4.1 (Busenberg and Cooke [7]). Suppose that

(i) there exist constants $p>0, q>0$ such that

$$
\eta_{B C}(t ; p):=2 p a(t)-b^{2}(t+h)-p^{2} \geq q \text { for all } t \in \mathbb{R}_{+},
$$

(ii) $\int_{t}^{t+h} b^{2}(s) d s$ is bounded, and

(iii) given $M>0$, there exists $\tau>0$ such that $\int_{t}^{t+\tau}|b(s)| d s<M$ for all $t \in \mathbb{R}_{+}$.

Then the zero solution of the linear equation

$$
x^{\prime}(t)=-a(t) x(t)+b(t) x(t-h)
$$

is $U A S$.

Let us apply Theorem 2.2 to the nonlinear generalization (4.1) of (4.5) with the Lyapunov functional

$$
V(t, \varphi):=|\varphi(0)|+\int_{t-h}^{t}|b(s+h)||g(\varphi(s-t))| d s .
$$

For every neighborhood of the origin there is a constant $c_{1}$ such that

$$
V(t, \varphi) \leq|\varphi(0)|+c_{1}\left(\int_{t}^{t+h} b^{2}(s) d s\right)^{1 / 2}\|\varphi\|_{2}
$$

for all $\varphi$ in the neighborhood and for all $t \in \mathbb{R}_{+}$. The derivative of $V$ with respect to (4.1) satisfies

$$
V_{[4.1]}^{\prime}\left(t, x_{t}\right) \leq-[a(t)-c|b(t+h)|]|f(x(t))| .
$$

From Theorem 2.2 we obtain

Corollary 4.2. Suppose that

(i) $\eta_{1}(t ; c):=a(t)-c|b(t+h)| \geq 0$ for all $t \in \mathbb{R}_{+}$, and

(ii) $\int_{t}^{t+h} b^{2}(s) d s$ is bounded on $\mathbb{R}_{+}$.

If $\int_{H} \eta_{1}(t ; c) d t=\infty$ for all $h$-dense sets $H \subset \mathbb{R}_{+}$, then the zero solution of (4.1) is $A S$. If, in addition, $\int_{\left[t_{0}, \infty\right) \cap H} \eta_{1}(t ; c) d t=\infty$ uniformly with respect to $t_{0} \in \mathbb{R}_{+}$and $h$-dense sets $H \subset \mathbb{R}_{+}$, then the zero solution of (4.1) is UAS.

Obviously, $\eta_{1}(t ; 1) \geq(1 / 2 p) \eta_{B C}(t ; p)$ for all $t \in \mathbb{R}_{+}, p>0$. Therefore, it follows from Corollary 4.2 that conditions (i)-(ii) in Theorem 4.1 alone imply UAS, i.e., condition (iii) can be dropped from Theorem4.1. Moreover, Corollary 4.2 sharpens Theorem 4.1 for both large and small $a(t), b(t)$ (e.g., while $a(t) \geq p / 2>0$ for all $t \in \mathbb{R}_{+}$in Theorem 4.1, Corollary 4.2 allows $a(t)$ to vanish on intervals of arbitrary lengths).

Finally, let us improve the conditions of three examples from Hale and Lunel [9].

Example 4.3 (Hale and Lunel [9]). Suppose that

(i) there are constants $p>0, q>0$ such that

$$
\eta_{H L}(t ; p):=(2 a(t)-p) p-b^{2}(t) \geq q \text { for all } t \in \mathbb{R}_{+} ;
$$

(ii) $a(t)$ and $b(t)$ are bounded on $\mathbb{R}_{+}$. 
Then the zero solution of 4.5 is UAS.

Example 4.4 (Hale and Lunel 9]). Suppose that

(i) there are constants $p>0, q \in(0,1)$ such that $a(t) \geq p$ and $|b(t)| \leq q p$ for all $t \in \mathbb{R}_{+}$;

(ii) $a(t)$ is bounded on $\mathbb{R}_{+}$.

Then the zero solution of the equation

$$
x^{\prime}(t)=-a(t) x^{3}(t)+b(t) x^{3}(t-h)
$$

is UAS.

Now we give a common generalization of these examples, sharpening their conditions at the same time. Choose $\nu(s)=p / 2$ in (4.2). Then (4.3) has the form

$V_{\text {[4.1] }}^{\prime}\left(t, x_{t}\right) \leq-\frac{1}{2 p} \eta_{2}(t ; p, c) f^{2}(x(t))(t \geq 0) ; \quad \eta_{2}(t ; p, c):=\left(2 a(t)-c^{2} p\right) p-b^{2}(t)$, and Theorem 2.2 yields

Corollary 4.5. Suppose that $\eta_{2}(t ; p, c) \geq 0$ for all $t \in \mathbb{R}_{+}$with some $p>0$. If $\int_{H} \eta_{2}(t ; p, c) d t=\infty$ for all $h$-dense sets $H \subset \mathbb{R}_{+}$, then the zero solution of [4.1) is $A S$. If, in addition, $\int_{\left[t_{0}, \infty\right) \cap H} \eta_{2}(t ; p, c) d t=\infty$ uniformly with respect to $t_{0} \in \mathbb{R}_{+}$ and $h$-dense sets $H \subset \mathbb{R}_{+}$, then the zero solution of (4.1) is $U A S$.

It is worth noticing that this corollary does not contain any boundedness condition on $a, b$.

Example 4.6 (Hale and Lunel [9]). Suppose that $a(t) \equiv a_{0}=$ const., $a_{0}>0$, and $b(t)$ is bounded on $\mathbb{R}_{+}$. Then the zero solution of the equation

$$
x^{\prime}(t)=-a(t) x^{3}(t)+b(t) x^{4}(t-h)
$$

is UAS.

Corollary 4.7. Suppose that there exists $p>0$ such that $a(t) \geq p|b(t+h)|$ and $\int_{t}^{t+h} b^{2}(s) d s$ is bounded for all $t \in \mathbb{R}_{+}$. If $\int_{H} a(t) d t=\infty$ for all $h$-dense sets $H \subset$ $\mathbb{R}_{+}$, then the zero solution of Equation (4.10) is $A S$. If, in addition, $\int_{\left[t_{0}, \infty\right) \cap H} a(t) d t$ $=\infty$ uniformly with respect to $t_{0} \in \mathbb{R}_{+}$and $h$-dense sets $H \subset \mathbb{R}_{+}$, then the zero solution of 4.10 ) is $U A S$.

Proof. From (4.6)-(4.8) it follows (see, e.g., 99 Th. 5.2.1]) that the zero solution of (4.10) is uniformly stable; therefore, we may suppose that $|x| \leq p / 2$. Then $\left|x^{4}\right| \leq(p / 2)\left|x^{3}\right|$, and taking into account also the identity

$$
a(t)-\frac{p}{2}|b(t+h)|=\frac{1}{2} a(t)+\frac{1}{2}[a(t)-p|b(t+h)|] \geq \frac{1}{2} a(t) \text { for } t \in \mathbb{R}_{+},
$$

we can apply Corollary 4.2 with $c=p / 2$.

4.2. Condition (2.10) cannot be replaced by (2.11). Condition (2.10) in Theorem 2.2 seems rather sophisticated in comparison with (2.11) in Theorem 2.3. The following example shows that this is well-founded in the sense that Theorem 2.2 with condition (2.11) instead of (2.10) would not be true. The construction of the example uses some ideas of [13, 22]. Introduce the function

$$
g(t)= \begin{cases}1+\frac{1}{2^{k+1}} & \text { if } k \leq t \leq k+1 / 2 \\ \frac{1}{2^{k+1}} & \text { if } k+5 / 8 \leq t \leq k+7 / 8, \quad k \in \mathbb{N},\end{cases}
$$


and extend this function so that $g: \mathbb{R}_{+} \rightarrow(0, \infty)$ is continuously differentiable on the whole half-line and monotone on the intervals $[k+1 / 2, k+5 / 8],[k+7 / 8, k+1]$ $(k \in \mathbb{N})$. Obviously, $g$ has the properties

$$
\begin{array}{ll}
g(t)-g(t+1) \geq 0 & \text { for all } t \in \mathbb{R}_{+}, \\
g(t)-g(t+1)=\frac{1}{2^{k+2}} & \text { if } k+\frac{5}{8} \leq t \leq k+\frac{7}{8} \quad(k \in \mathbb{N}) .
\end{array}
$$

Denote

$$
\alpha_{k}:=\frac{g(k+1)}{g(k)}=\frac{2^{k+2}+1}{2^{k+2}+2} ; \quad p_{k}:=\frac{\alpha_{k}}{1-\alpha_{k}}=2^{k+2}+1 \quad(k \in \mathbb{N}) .
$$

Let us define a function $f: \mathbb{R}_{+} \times C([-1,0] ; \mathbb{R}) \rightarrow \mathbb{R}$ by the following definitions:

a) If $\varphi(0) \geq 0$, and $k \leq t<k+1$ for some $k \in \mathbb{N}$, then

$$
f(t, \varphi):= \begin{cases}\frac{g^{\prime}(t)}{g(t)} \varphi(0) & \text { if } k \leq t \leq k+7 / 8, \\ p_{k} \frac{g^{\prime}(t)}{g(t)}\left(\|\varphi\|_{\infty}-\varphi(0)\right) & \text { or } \varphi(0) \leq \alpha_{k}\|\varphi\|_{\infty}, \\ & \text { and } \varphi(0)>\alpha_{k}\|\varphi\|_{\infty} .\end{cases}
$$

b) If $\varphi(0)<0$, then

$$
f(t, \varphi):=-f(t,-\varphi) \text {. }
$$

We prove that $f$ is continuous. In fact, if $k \leq t \leq k+1$ and $0<\varphi(0)=\alpha_{k}\|\varphi\|_{\infty}$, then

$$
\begin{aligned}
f(t, \varphi)=\frac{g^{\prime}(t)}{g(t)} \varphi(0) & =\frac{g^{\prime}(t)}{g(t)} \alpha_{k}\|\varphi\|_{\infty}=p_{k} \frac{g^{\prime}(t)}{g(t)}\left(1-\alpha_{k}\right)\|\varphi\|_{\infty} \\
& =p_{k} \frac{g^{\prime}(t)}{g(t)}\left(\|\varphi\|_{\infty}-\varphi(0)\right) .
\end{aligned}
$$

On the other hand, $f(k, \varphi)=0$ for all $k \in \mathbb{N}, \varphi \in C$, and $f(t, \varphi)=0$ if $\varphi(0)=0$ and $t \in \mathbb{R}_{+}$; therefore, $f$ is continuous, and what is more, it is locally Lipschitz.

Now consider the functional differential equation

$$
x^{\prime}(t)=f\left(t, x_{t}\right) .
$$

We prove that for every $\left(t_{0}, \psi\right) \in \mathbb{R}_{+} \times C([-1,0] ; \mathbb{R})$ there exists a constant $c$ such that $|c| \leq\|\psi\|_{\infty}$ and the solution $x\left(t_{0}, \psi\right)$ of Equation (4.12) satisfies the conditions

$$
\begin{aligned}
x\left(t ; t_{0}, \psi\right) & =c g(t) & & \text { for } t \geq\left[t_{0}\right]+1, \\
\left|x\left(t ; t_{0}, \psi\right)\right| & \leq\|\psi\|_{\infty} & & \text { for all } t \geq t_{0},
\end{aligned}
$$

where $[\cdot]$ denotes the greatest integer function. We prove (4.13) and (4.14) for $\psi(0) \geq 0$; the case $\psi(0)<0$ is similar.

First we show that $k \leq t_{0} \leq k+1 / 2$ implies

$$
x(t)=x\left(t ; t_{0}, \psi\right)=\frac{\psi(0)}{g\left(t_{0}\right)} g(t) \quad\left(t \geq t_{0}\right) .
$$

For $t \in\left[t_{0}, k+7 / 8\right]$ this is obviously satisfied by the definition of the right-hand side $f$. If (4.15) is not true for some $t \in(k+7 / 8, k+1]$, then introduce

$$
t_{*}:=\inf \left\{t \in(k+7 / 8, k+1]: x(t)>\alpha_{k}\left\|x_{t}\right\|_{\infty}\right\} .
$$


We have $t_{*}<k+1$ and $x(t) \leq \alpha_{k}\left\|x_{t}\right\|_{\infty}$ for $t \in\left[k+7 / 8, t_{*}\right]$; therefore,

$$
x\left(t_{*}\right)=\frac{\psi(0)}{g\left(t_{0}\right)} g\left(t_{*}\right)<\frac{g(k+1)}{g(k)} \psi(0)=\alpha_{k} \psi(0) \leq \alpha_{k}\left\|x_{t_{*}}\right\|_{\infty},
$$

i.e., $x\left(t_{*}\right)<\alpha_{k}\left\|x_{t_{*}}\right\|_{\infty}$, which contradicts the definition of $t_{*}$. So (4.15) is true on $\left[t_{0}, k+1\right]$ and, consequently, for all $t \geq t_{0}$. Therefore, 4.13) holds with $c=$ $\psi(0) / g\left(t_{0}\right)$, whence (4.14) follows.

If $k+1 / 2<t_{0}<k+1$, then, in general, we have no formula for $x\left(t_{0}, \psi\right)$, but we know that $\left\|x_{t}\right\|_{\infty} \leq\|\psi\|_{\infty}\left(t \in\left[t_{0}, k+1\right]\right)$. This follows from the fact that a $t^{*}$ is an increasing point of the function $t \mapsto\left\|x_{t}\right\|_{\infty}$ only if it is an increasing point of $t \mapsto x(t)$, and $x\left(t^{*}\right)=\left\|x_{t^{*}}\right\|_{\infty}$, but this is impossible by the definition of $f$. This means that (4.13) and (4.14) are satisfied with $c:=x\left(k+1 ; t_{0}, \psi\right) / g(k+1)$.

Now consider the functionals

$$
\begin{gathered}
V_{1}(\varphi):=\max \{|\varphi(0)| ;|\varphi(-1 / 2)|\}, \quad V_{2}(\varphi):=\|\varphi\|_{2}^{2}, \\
V(\varphi):=V_{1}(\varphi)+V_{2}(\varphi) \quad(\varphi \in C([-1,0] ; \mathbb{R})) .
\end{gathered}
$$

We prove that along every solution $x(t)=x\left(t ; t_{0}, \psi\right)$ of Equation 4.12$)$ the following conditions are satisfied:

(i) $|x(t)| \leq V\left(x_{t}\right) \leq\|\psi\|_{\infty}+\|\psi\|_{\infty}^{2}$ for $t \geq t_{0}$;

(ii) $V\left(x_{t}\right) \leq \max \{|x(t)| ;|x(t-1 / 2)|\}+\left\|x_{t}\right\|_{2}^{2}$ for $t \geq t_{0}$;

(iii) $V^{\prime}\left(t, x_{t}\right) \leq 0$ for $t \geq\left[t_{0}\right]+2$;

(iv) $V^{\prime}\left(t, x_{t}\right) \leq-\eta(t) x^{2}(t)$ for $t \geq\left[t_{0}\right]+2$, where

$$
\eta(t):= \begin{cases}2 & \text { if } t \in \bigcup_{k \in \mathbb{N}}\left[k+\frac{5}{8}, k+\frac{7}{8}\right], \\ 0 & \text { otherwise. }\end{cases}
$$

In fact, (i) and (ii) follow from (4.14) and the definition of $V$. Property (4.13) implies that the function $t \mapsto V_{1}\left(x_{t}\right)$ is nonincreasing for $t \geq\left[t_{0}\right]+2$. Furthermore, if $k+5 / 8 \leq t \leq k+7 / 8$ for some $k \in \mathbb{N}$, then, by (4.11), we have

$$
\begin{aligned}
V_{2}^{\prime}\left(t, x_{t}\right) & =x^{2}(t)-x^{2}(t-1)=-c^{2}\left(g^{2}(t-1)-g^{2}(t)\right) \\
& =-c^{2}(g(t-1)-g(t))(g(t-1)+g(t)) \\
& \leq-c^{2} \frac{1}{2^{k+1}} 2 g(t)=-2 c^{2} g^{2}(t)=-2|x(t)|^{2},
\end{aligned}
$$

whence (iv) follows.

Now we can state:

1. The zero solution of Equation (4.12) is not AS (see property (4.13) and the definition of $g$ ).

2. All conditions but 2.10) in Theorem 2.2 are satisfied (see properties (i)-(iv) and Remark 3.2).

3. Instead of condition (2.10) we have

$$
\lim _{K \rightarrow \infty} \int_{t_{0}}^{t_{0}+K} \eta(t) d t=\infty
$$

i.e., condition (2.11) in Theorem 2.3.

This means that Theorem 2.2 would not be true with condition (2.11) instead of (2.10). 
4.3. Theorems 2.2 and 2.3 are independent. Estimate (4.3) is an example for the situation when Theorem 2.2 is applicable, but Theorem 2.3 is not. Now we construct an example for the opposite situation.

Let us consider the linear FDE with one delay

$$
x^{\prime}(t)=-a(t) x(t)+b(t) x(t-2),
$$

where

$$
\begin{aligned}
& a(t):= \begin{cases}1 & \text { if } t \in \bigcup_{k \in \mathbb{N}}[2 k, 2 k+1], \\
0 & \text { otherwise }\end{cases} \\
& b(t):= \begin{cases}1 & \text { if } t \in \bigcup_{k \in \mathbb{N}}[8 k+2,8 k+3], \\
0 & \text { otherwise }\end{cases}
\end{aligned}
$$

and choose the Lyapunov functional (4.6) with $g(x) \equiv x$. Then we have estimate (4.8) with $f(x) \equiv x, c=1$, and $h=2$. However, now Theorem 2.2 cannot be applied because (2.10) is not satisfied. On the other hand, we have the identity

$$
|x(t)|=\exp \left[-\int_{t-2}^{t} a(s) d s\right]|x(t-2)|
$$

for $t \in[8 k-3,8 k+2]$ and the inequality

$$
V_{\text {(4.16) }}^{\prime}\left(t, x_{t}\right) \leq-[a(t)-|b(t+2)|]|x(t)|=-a(t)|x(t)|
$$

for $t \in[8 k+1,8(k+1)](k \in \mathbb{N})$. Therefore,

$$
\begin{aligned}
V_{\text {(4.16) }}^{\prime}\left(t, x_{t}\right) & \leq-a(t)|x(t)|=-a(t) \exp \left[-\int_{t-2}^{t} a(s) d s\right]|x(t-2)| \\
& \leq-\frac{1}{e \sqrt{2}}\left\|x_{t}\right\|_{2} \quad \text { for } t \in[8 k+6,8 k+7] \quad(k \in \mathbb{N}) .
\end{aligned}
$$

Consequently, Theorem 2.3 guarantees UAS for the zero solution of (4.16).

\section{ACKNOWLEDGMENT}

The author would like to thank the referees for a careful reading of the paper and for a good number of suggestions.

\section{REFERENCES}

1. A. S. Andreev, On the stability of a nonautonomous functional-differential equation. Dokl. Akad. Nauk 356 (1997), 151-153. (Russian) MR 98m:34149

2. Z. S. Athanassov, Families of Liapunov-Krasovski乞 functionals and stability for functional differential equations. Ann. Mat. Pura Appl. (4) 176 (1999), 145-165. MR 2000m:34164

3. T. A. Burton, Uniform asymptotic stability in functional differential equations. Proc. Amer. Math. Soc. 68 (1978), 195-199. MR 58:1489

4. T. A. Burton and L. Hatvani, Stability theorems for nonautonomous functional-differential equations by Liapunov functionals. Tôhoku Math. J. (2) 41 (1989), 65-104. MR 90d:34147

5. T. A. Burton and L. Hatvani, On nonuniform asymptotic stability for nonautonomous functional-differential equations. Differential Integral Equations 3 (1990), 285-293. MR 90k:34098

6. T. A. Burton and G. Makay, Asymptotic stability for functional-differential equations. Acta Math. Hungar. 65 (1994), 243-251. MR 95d:34126

7. S. N. Busenberg and K. L. Cooke, Stability conditions for linear nonautonomous delay differential equations. Quart. Appl. Math. 42 (1984), 295-306. MR 85j:34156

8. I. V. Gă̌shun and L. B. Knyazhishche, Nonmonotone Lyapunov functionals. Conditions for the stability of equations with delay. Differential Equations 30 (1994), 1195-1200. MR 96g:34118 
9. J. K. Hale and S. M. Verduyn Lunel, Introduction to functional-differential equations. Springer-Verlag, New York, 1993. MR 94m:34169

10. L. Hatvani, On the asymptotic stability of the solutions of functional-differential equations. Qualitative theory of differential equations (Szeged, 1988), North-Holland, Amsterdam, 1990, pp. 227-238. MR 92f:34072

11. L. Hatvani, On the asymptotic stability for functional differential equations by Lyapunov functionals. Nonlinear Anal. (Ser. A: Theory Methods) 40 (2000), 251-263. MR 2001c:34158

12. L. Hatvani, Annulus arguments in the stability theory for functional differential equations. Differential Integral Equations 10 (1997), 975-1002. MR 2000m:34169]

13. J. Kato, A conjecture in Lyapunov method for functional-differential equations. World Congress of Nonlinear Analysts 1992, Vols. I-IV (Tampa, FL, 1992), de Gruyter, Berlin, 1996, pp. 1239-1246. MR 97a:00029

14. Y. Ko, An asymptotic stability and a uniform asymptotic stability for functional-differential equations. Proc. Amer. Math. Soc. 119 (1993), 535-545. MR 93k:34166

15. K. Kobayashi, Asymptotic stability in functional-differential equations. Nonlinear Anal. 20 (1993), 359-364. MR 93m:34127

16. V. B. Kolmanovskiŭ, L. Torelli, and R. Vermiglio, Stability of some test equations with delay. SIAM J. Math. Anal. 25 (1994), 948-961. MR 95c:34135

17. N. N. Krasovskiu, Stability of motion. Applications of Lyapunov's second method to differential systems and equations with delay. Stanford University Press, Stanford, Calif., 1963. MR 26:5258

18. V. Lakshmikantham, S. Leela, and A. A. Martynyuk, Stability analysis of nonlinear systems. Marcel Dekker Inc., New York, 1989. MR 90h:34073

19. V. Lakshmikantham, S. Leela, and S. Sivasundaram, Liapunov functions on product spaces and stability theory of delay differential equations. J. Math. Anal. Appl. 154 (1991), 391-402. MR 92a:34086

20. X. Liu and D. Y. Xu, Uniform asymptotic stability of abstract functional-differential equations. J. Math. Anal. Appl. 216 (1997), 626-643. MR 98j:34159

21. G. Makay, On the asymptotic stability in terms of two measures for functional-differential equations. Nonlinear Anal. 16 (1991), 721-727. MR 92b:34093

22. G. Makay, An example on the asymptotic stability for functional-differential equations. Nonlinear Anal. 23 (1994), 365-368. MR 95i:34147

23. G. Makay, On the asymptotic stability of the solutions of functional-differential equations with infinite delay. J. Differential Equations 108 (1994), 139-151. MR 95d:34130

24. J. Terjéki, Power-asymptotic stability for functional-differential equations. The Lyapunov functions method and applications, Baltzer, Basel, 1990, pp. 161-164. MR 92i:34003

25. T. X. Wang, Stability in abstract functional-differential equations. I. General theorems. J. Math. Anal. Appl. 186 (1994), 534-558. MR 95k:34112

26. T. X. Wang, Stability in abstract functional-differential equations. II. Applications. J. Math. Anal. Appl. 186 (1994), 835-861. MR 96a:34155

27. B. Zhang, Asymptotic stability in functional-differential equations by Liapunov functionals. Trans. Amer. Math. Soc. 347 (1995), 1375-1382. MR 95g:34116

Bolyai Institute, University of Szeged, Aradi vértanúk tere 1, H-6720 Szeged, HunGARY

E-mail address: hatvani@math.u-szeged.hu 